\title{
Connectivity of the Superior Longitudinal Fasciculus II and Executive Function in Children with ADHD
}

\author{
Larissa Ma ${ }^{1}$, Leya Joykutty"${ }^{1 \#}$ and Anthony Dick ${ }^{\#}$ \\ ${ }^{1}$ American Heritage School, Plantation, FL, USA \\ \#Advisor
}

$\underline{\text { ABSTRACT }}$

Attention-deficit hyperactivity disorder (ADHD) is a common childhood neurodevelopmental disorder marked by inattention, hyperactivity, and impulsiveness. The importance of the Superior Longitudinal Fasciculus II (SLFII), a white matter tract connecting the frontal and parietal regions, to developing executive function has not been established in children who typically have executive function deficits. The present study explored the association between diffusion properties of the SLFII and developing executive function in young children diagnosed with ADHD. A dataset with the performance on the NIH Toolbox Card Sort and the NIH Toolbox Flanker, two executive function tasks, in 59 4-7-year-old children was used, and diffusion-weighted magnetic resonance imaging scans were used to quantify the white matter properties of the bilateral SLFII. The results showed that there was an association between age and performance on the Flanker and Card Sort tasks. As age increased, performance also improved. There was no significant association between white matter properties of the SLFII, birth sex, and the Card Sort and Flanker tasks. As the first study to explore this association in children of this age with ADHD, this result was unexpected suggesting that it may be the case that it is too early in development to detect a strong association. The findings inform contemporary and future investigations into the brain-behavior relations between SLFII and executive function in children with ADHD. With further research, neuroimaging could become a potential diagnostic biomarker for predicting executive function impairments and ADHD symptoms in young children, potentially altering treatment outcomes.

\section{Introduction}

It is well-established that the frontal and parietal regions of the human brain are involved in the organism's ability to organize, and control directed voluntary behavior (Alvarez \& Emory, 2006; Fischer et al., 2020). These are the socalled executive functions, which are defined as higher-level cognitive functions involved in the control and regulation of "lower-level" cognitive processes and voluntary behaviors (Alvarez \& Emory, 2006). They are typically measured using laboratory-based tasks, such as Card-Sorting or Flanker tasks, which require the participant to switch attention to certain aspects of a visual stimulus (in the case of Card Sort tasks), or otherwise resist interference to distracting stimuli (in the case of the Flanker task; Zelazo et al., 2013). Several studies have suggested that the parietal and frontal lobes are critical to satisfactory performance on these tasks (Roberts et al., 2010; Berman, 1995)

Researchers have also become interested in the white matter connections that support communication between these distant brain regions in the human cortex. For example, research in non-human primates has shown that the connection between middle and superior frontal and superior parietal brain regions is achieved by the superior longitudinal fasciculus II (SLFII), in both the left and right hemispheres (Schmahmann \& Pandya, 2006). This has been validated in humans using a non-invasive technique diffusion-weighted magnetic resonance imaging (DW-MRI). In this technique, the diffusion of water in the brain is measured while a participant is undergoing an MRI. Because water diffuses directional down axons in the brain, the diffusion of water in axons can be directly measured. These axons form bundles in the brain, which are termed fasciculi. Each of these several fasciculi, for example the SLFII, can be tracked in acquired DW-MRI scans. The integrity of the fiber pathway can then be interrogated using this 
method, such that greater integrity of a given fiber pathway is associated with more constrained diffusion of water (Catani \& Thiebaut de Schotten, 2008).

Using this technique, researchers have established the importance of the SLFII for supporting executive functions in the human brain. For example, studies have shown a significant positive association between the SLF II and common executive function in young adults (Smolker et al., 2018). A middle network associated with the SLF II was activated in both spatial/motor functions and non-spatial/motor functions (Parlatini et al., 2017). In patients who underwent prefrontal glioma resection and patients who did not, there was significant correlation between spatial working memory deficit and the region around the SLF II (Kinoshita et al., 2016). In addition, the SLF II was associated with the strategy score- a measure of executive function. This shows the importance of the SLFII fiber pathway for executive function.

\section{Review of Literature}

Although these studies have been conducted in adults, the importance of the SLFII to developing executive function has not been established, in particular in children who typically have executive function deficits. Children with attention-deficit hyperactivity disorder (ADHD) are much more likely than typical children to show executive function deficits (Mullane et al., 2008). No study has examined whether the association between SLFII integrity and executive function is apparent in these children. It may be the case that the developing connectivity between frontal and parietal regions is disrupted or altered in children with ADHD. This is what we aimed to establish in the present study.

Thus, for the present study we used de-identified data from a sample of young children diagnosed with ADHD who were then scanned using DW-MRI in an MRI machine. Their executive function performance was measured using two executive function tasks, the NIH Toolbox Card Sort and the NIH Toolbox Flanker. We 1) tracked the SLFII using the DW-MRI scan in each subject for each hemisphere and 2) related the integrity of the SLFII white matter to performance on the two executive function tasks. In this way we aimed to establish whether there is an association between development in this pathway and executive function in young children who typically show executive function deficits.

\section{Methods}

\section{Dataset}

The data was taken from a large urban southeastern city in the U.S. with a large Latinx population. Children and their caregivers were recruited from local preschools and mental health agencies via brochures, radio and newspaper ads, and open houses/parent workshops. For the ADHD sample, parents and children were invited to participate in an assessment to determine study eligibility if the parent (1) endorsed his or her child as having clinically significant levels of ADHD symptoms, (2) indicated that his or her child was currently displaying clinically significant academic, behavioral, or social impairments as measured by a score of three or higher on a seven-point impairment rating scale (Fabiano et al., 2006), and (3) indicated that his or her child was not taking any psychotropic medication. Participants were also required to be enrolled in school during the previous year, have an estimated IQ of 70 based on the WPPSIIV (Wechsler, 2012), have no confirmed history of an Autism Spectrum Disorder, and be able to attend an 8-week summer treatment program (STP-PreK; Graziano, Slavec, Hart, Garcia, and Pelham (2014) prior to the start of the next school year.

ADHD diagnosis and comorbid disruptive behavior disorders were assessed through a combination of parent structured interview (Computerized-Diagnostic Interview Schedule for Children [C-DISC]; (Shaffer, Fisher, Lucas, Dulcan, \& Schwab-Stone, 2000) and parent and teacher ratings of symptoms and impairment (Disruptive Behavior 
Disorders Rating Scale, Impairment Rating Scale; Fabiano et al., 2006; Pelham, Gnagy, Greenslade, \& Milich, 1992), as is recommended practice. Dual Ph.D. level clinician review was used to determine diagnosis and eligibility.

The final participating sample consisted of 59 young children $\left(M_{\text {age }}=5.58, S D=0.76\right.$, and $73 \%$ male reflecting the higher incidence of males in ADHD). Eighty percent of the children were identified by parents as Hispanic/Latino White, $12 \%$ as Non-Hispanic/Latino White, $6 \%$ as Non-Hispanic/Latino Black, and $2 \%$ as Hispanic/Latino Black. All subjects were de-identified prior to data analysis.

\section{Measurement of Executive Function}

Executive function was measured using two tasks: the NIH Toolbox Dimensional Change Card Sort (DCCS) to measure task-switching/cognitive flexibility (Zelazo, Anderson, Richler, Wallner-Allen, Beaumont, \& Weintraub, 2013), and the NIH Toolbox Flanker Inhibitory Control and Attention Test (NIH Toolbox Flanker).

The NIH Toolbox "card sort" task is based on the DCCS developed by Zelazo and colleagues (Zelazo et al., 2013). In the standard version of the DCCS, children are shown two target cards (e.g., red rabbits and blue boats) and asked to sort test cards (e.g., blue rabbits and red boats) first according to one dimension (e.g., color), and then according to the other (e.g., shape). Because of the conflict between the target and test cards, switching between the first rule and the second incurs a switch cost, both in terms of accuracy and response time (RT). In the NIH Toolbox version, there are four card sorting blocks: practice, preswitch, postswitch, and mixed. In the practice block, participants were instructed to match centrally presented stimuli to one of two lateralized target stimuli. The preswitch and postswitch trials were similar to the practice trials, but there was a conflict between the test and target cards. A rule switch was employed between the pre- and postswitch phase, with the sorting dimension (shape or color) counterbalanced across participants. Children who succeeded on at least four trials of the postswitch received the mixed block, which consisted of 50 trials of 40 "frequent" and 10 "infrequent" trials. The frequent trials corresponded to the dimension that had been presented in the postswitch phase. The standard toolbox scoring was used, in which both accuracy and RT are included in the score (Zelazo et al., 2013).

The NIH Toolbox Flanker was administered to the participants in the sample in the standard fashion. In this task, a version of the Eriksen flanker task (Eriksen \& Eriksen,1974), participants are required to indicate the left-right orientation of a centrally presented stimulus while inhibiting attention to the "flankers" that surround it on each side. On some trials, orientation of the flankers is congruent with the orientation of the central stimulus; on other trials it is incongruent. The NIH Toolbox version consists of a practice block, a block using child-friendly fish stimuli, and a block using more difficult arrow stimuli. Scoring is similar to the Card Sort scoring, and incorporates both accuracy and RT.

\section{MRI Acquisition \& Processing}

All imaging was performed using a research-dedicated 3 Tesla Siemens MAGNETOM Prisma MRI scanner (V11C) with a 32-channel coil located on the university campus. Children first completed a preparatory phase using a realistic mock scanner. In the magnet children watched a child-friendly movie of their choice. Ear protection was used, and sound was presented through MRI compatible headphones.

We collected multi-shell high-angular diffusion-weighted imaging (HARDI) data according to the Adolescent Brain and Cognitive Development (ABCD) protocol (Hagler et al., 2019). These scans were collected with a 1.7 $\mathrm{mm}$ isotropic voxel size, using multiband imaging echo planar imaging (EPI; acceleration factor $=3$ ). The acquisition consisted of ninety-six diffusion directions, six $b=0$ frames, and four $b$-values (102 diffusion directions; $6 \mathrm{~b}=500,15$ $b=1000,15 b=2000$, and $60 b=3000$ ).

\section{Diffusion-weighted Imaging Post-Processing}

Initial post-processing was accomplished with DTIPrep v1.2.8 (Oguz et al., 2014), TORTOISE DIFFPREP v3.1.0 (Irfanoglu, Nayak, Jenkins, \& Pierpaoli, 2017; Pierpaoli et al., 2010), FSL v6.0.1 topup (Andersson, Skare, \& 
Ashburner, 2003; Smith et al., 2004), and DSI Studio (v. June 2020; Yeh, Wedeen, \& Tseng, 2010). We also implemented a pre- and post-analysis quality check assessing signal-to-noise of each diffusion b-value (Roalf et al., 2016).

Initial quality control was accomplished in DTIPrep to complete the following steps: 1) image/diffusion information check; 2) padding/cropping of data; 3) Rician noise removal; 4) slice-wise, interlace-wise, and gradientwise intensity and motion checking. The number of acquisitions removed was used as a proxy for movement/bad data quality, and was included as a covariate in subsequent regression analyses. TORTOISE DIFFPREP was used to accomplish motion and eddy current correction. We implemented calculation of the diffusion tensor model in DSI Studio to estimate the eigenvalues reflecting diffusion parallel and perpendicular to each of the fibers along three axes (x, $\mathrm{y}$, z). In addition to calculating the more familiar diffusion metrics, we also reconstructed the data using higher-order HARDI generalized q-sampling imaging (GQI) technique (Yeh et al., 2010), implemented in DSI Studio. We calculated the Generalized Fractional Anisotropy (GFA) from this reconstruction, and this is our main predictive measure of interest. GFA can be thought of as a higher-order generalization of FA (Tuch, 2004). Like the traditional FA metric from diffusion tensor imaging, the GFA values range from 0 to 1 , with higher values associated with greater white matter integrity.

\section{Fiber Tract Identification}

Tractography was conducted using DSI Studio by manually reconstructing the left and right superior longitudinal fasciculus II in each subject, with reference to established connections in non-human primates that show a frontalparietal pathway in the superior white matter of the cortex (Schmahmann \& Pandya, 2006; see Figure 1). To do this, in the axial plane we identified a superior bundle of anterior-posterior directed streamlines (coded green in the directionally encoded brain image). We manually outlined this bundle to define the region of interest (ROI). We then set the tracking function to seed, and identified streamlines that connected the middle frontal cortex in the anterior course of the tract, and the intraparietal sulcus in the posterior course of the tract. This was done for each hemisphere and for each participant. As a final step, for each fiber pathway of interest, for each hemisphere, and for each subject, GFA statistics were exported and averaged across the whole fiber bundle for further analysis.

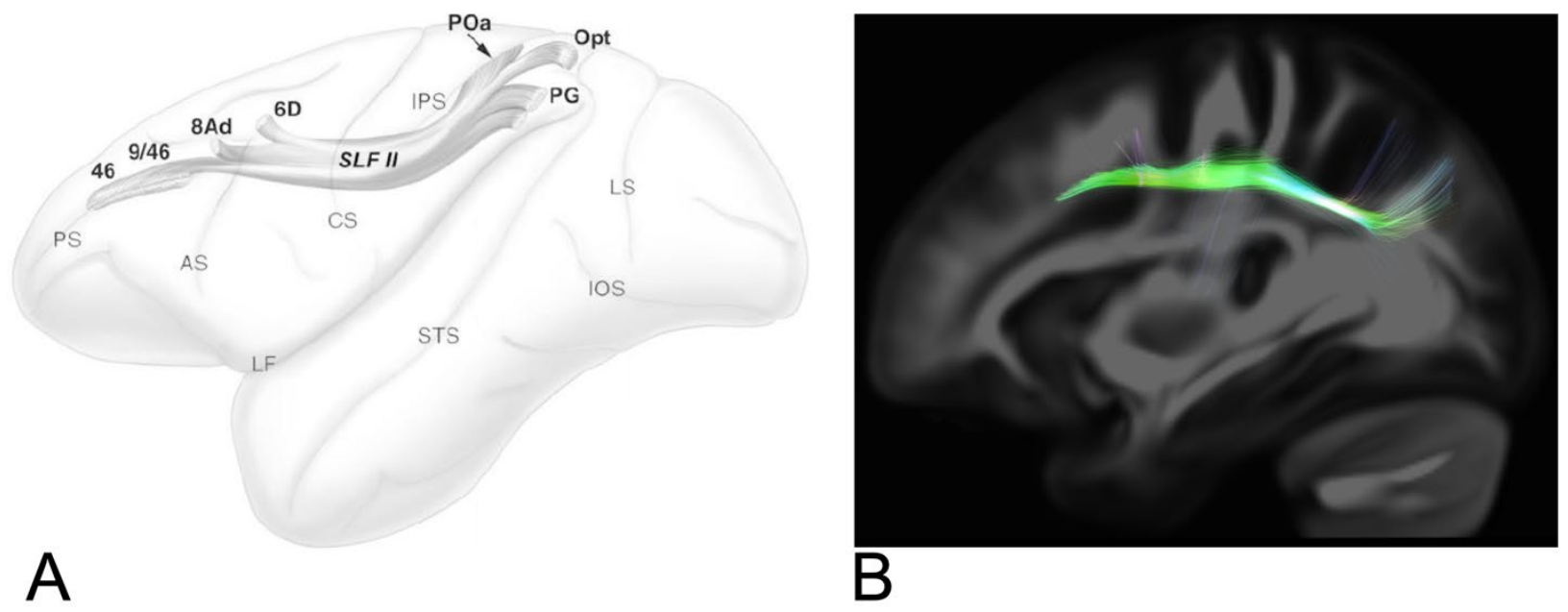

Figure 1. The superior longitudinal fasciculus II from fiber tracking autoradiography shown in a macaque monkey. B. Example subject showing the fiber tracking of the superior longitudinal fasciculus II in a diffusion-weighted imaging magnetic resonance imaging scan. Both images show the pathway connecting the middle and superior frontal and superior parietal lobes. 


\section{Brain-Behavior Data Analyses}

Analyses were conducted using R v.3.5.3 (Team, 2020) and used ordinary least squares regression. We also calculate and report the $95 \%$ Confidence Intervals (CIs). Four regressions were conducted: ( 1 and 2 ) right and left SLFII GFA entered as the independent variable and NIH Toolbox Card Sort as the dependent variable; and (3 and 4) right and left SLFII GFA entered as the independent variable and NIH Toolbox Flanker as the dependent variable. For each analysis, age and birth sex were entered as covariates of non-interest.

\section{Results}

Four separate regression analyses were conducted. The first two examined the association between the left and right SLFII GFA and NIH Toolbox Card Sort performance, controlling for age and birth sex. As Table 1 shows, only the association between age and performance was significant. The positive slope suggests that as age increased, performance on the Card Sort task also improved, and this association was better than that expected by chance (i.e., it was statistically significant). The second two analyses examined the association between the left and right SLFII GFA and NIH Toolbox Flanker performance, controlling for age and birth sex. As Table 2 shows, only the association between age and performance was significant. Again, the positive slope suggests that as age increased, performance on the Flanker task also improved, and this association was better than that expected by chance (i.e., it was statistically significant).

Table 1. Results of Regression Analysis for NIH Toolbox Card Sort

\begin{tabular}{|l|l|l|l|l|}
\hline & Estimate & Std. Error & t value & $\operatorname{Pr}(>|\mathbf{t}|)$ \\
\hline (Intercept) & 12.8088 & 20.9196 & 0.61 & 0.5428 \\
\hline Left SLFII & -45.3771 & 184.1972 & -0.25 & 0.8063 \\
\hline Age & 8.1998 & 2.9878 & 2.74 & $0.0081^{* *}$ \\
\hline Sex & -1.5596 & 4.9577 & -0.31 & 0.7542 \\
\hline & & & & \\
\hline (Intercept) & 2.7544 & 21.0801 & 0.13 & 0.8965 \\
\hline Right SLFII & 30.4128 & 154.4116 & 0.20 & 0.8445 \\
\hline Age & 9.2177 & 2.6474 & 3.48 & $0.0009^{* * *}$ \\
\hline Sex & -2.2791 & 4.7052 & -0.48 & 0.6298 \\
\hline
\end{tabular}

Note. A p-values are two-tailed. ${ }^{*} \mathrm{p}<.05 .{ }^{* *} \mathrm{p}<.01 .{ }^{* * *} \mathrm{p}<.001$.

Table 2. Results of Regression Analysis for NIH Toolbox Flanker

\begin{tabular}{|l|l|l|l|l|}
\hline & Estimate & Std. Error & t value & $\operatorname{Pr}(>|\mathbf{t}|)$ \\
\hline (Intercept) & -14.6975 & 22.6755 & -0.65 & 0.5195 \\
\hline Left SLFII & 40.2862 & 199.6581 & 0.20 & 0.8408 \\
\hline Age & 11.1630 & 3.2386 & 3.45 & $0.0011^{* *}$ \\
\hline Sex & 0.9661 & 5.3739 & 0.18 & 0.8580 \\
\hline & & & & \\
\hline (Intercept) & -9.3046 & 22.7800 & -0.41 & 0.6843 \\
\hline Right SLFII & -98.7859 & 166.8630 & -0.59 & 0.5560 \\
\hline Age & 13.1784 & 2.8609 & 4.61 & $0.0000^{* * *}$ \\
\hline Sex & -1.7159 & 5.0846 & -0.34 & 0.7369 \\
\hline
\end{tabular}

Note. A p-values are two-tailed. ${ }^{*} \mathrm{p}<.05 .{ }^{* *} \mathrm{p}<.01 .{ }^{* * *} \mathrm{p}<.001$. 


\section{SLF II Predicting NIH Toolbox Card Sort Scores}

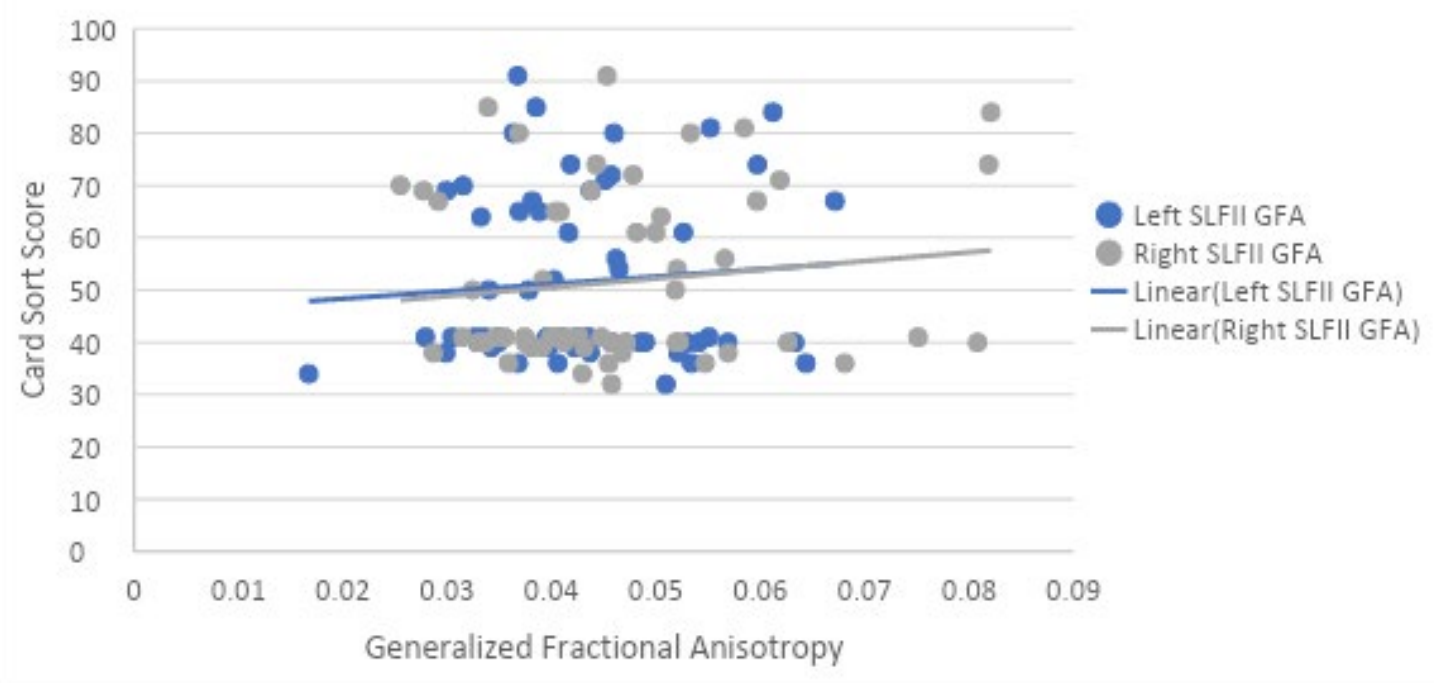

SLF II Predicting NIH Toolbox Flanker Scores

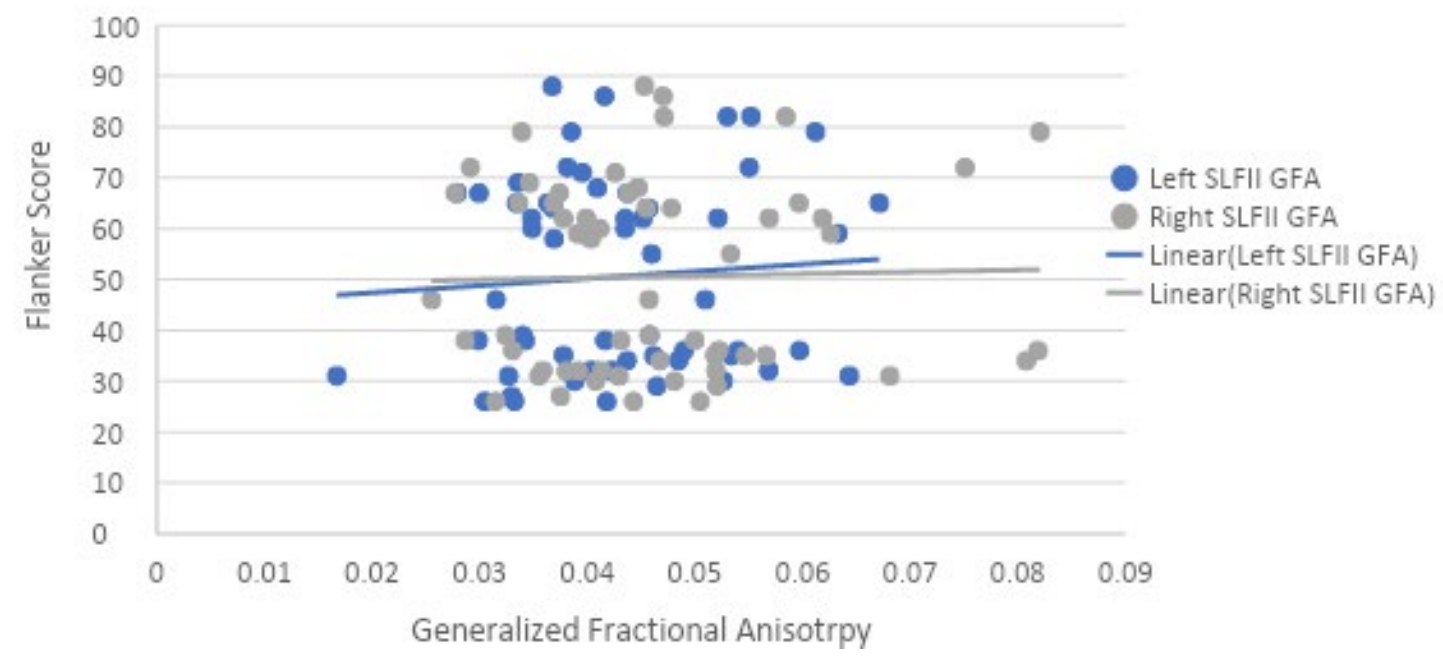

Figure 2. Scatterplots showing the association between generalized fractional anisotropy of the superior longitudinal fasciculus II and NIH Toolbox Card Sort and NIH Toolbox Flanker performance, for each hemisphere. The slopes of the regression lines were not significantly different from zero.

\section{Discussion}

In the present study we explored the association between diffusion properties of the SLFII and developing executive function in young children diagnosed with ADHD. The SLFII was chosen because of its important role in supporting connectivity between the frontal and parietal cortices on both hemispheres of the brain. These brain regions are known to support developing executive function and working memory skills in young children, and also to support these cognitive functions in adults. In young children with ADHD, executive function and working memory are typically delayed or impaired relative to typically developing children. Therefore, we expected the structure of the SLFII to be associated with executive function in young children with ADHD, particularly on two tasks which assess cognitive flexibility, attention, and resistance to interference from distracting stimuli. These two tasks were the NIH Toolbox 
Card Sort and the NIH Toolbox Flanker task. Thus, we assessed the performance on these tasks in 59 4-7-year-old children and associated this performance with the white matter properties of the bilateral SLFII as measured by DWMRI.

We found no association between white matter properties of either the left or right SLFII, or birth sex, and the Card Sort and Flanker tasks. We did find an association between age and performance on both the Card Sort and Flanker task, such that as age increased, performance increased. These findings are discussed below as it relates to understanding brain development of fiber pathways supporting executive function in young children with ADHD.

\section{Conclusion and Implications}

As we report above, we found no association between SLFII white matter and performance on the Flanker and Card Sort tasks. This was unexpected given that the fiber pathway has been associated with executive function and working memory in adults. For example, prefrontal glioma resection, particularly on the right hemisphere, is associated with working memory and executive function deficits when it impacts the white matter of the SLFII (Kinoshita et al., 2016). This association has never been investigated in children of this age, nor in children with ADHD. Our data, in finding no brain-behavior association, are inconsistent with the adult findings. However, two factors limit the generalizability of our reported findings. First, the sample size we investigated was reasonable, but still small from a statistical perspective. In cases where the effect of interest is difficult to detect or is also small, a smaller sample severely limits the ability to find an association better than chance. However, the sample size was sufficient to detect the effects of age. Thus, a second possibility is that the frontal and parietal brain regions are, during this time period of development, actually organizing to communicate and support the cognitive functions under investigation. That is, it may be the case that it is too early in development to detect a strong association. Further studies would need to be conducted to determine whether this is the case. Of course, lastly, it is also possible that there is no meaningful association, and therefore one was not detected.

We did find an association between age and both Card Sort and Flanker performance. Associations with age and improved performance on executive function tasks is well-established. For example, Zelazo and colleagues (2003; 2013) found that Card Sort performance improves substantially in children in this age range. Oeri and colleagues (2018) found the same association between age and Flanker performance, such that Flanker performance increases with increasing age in children (Mullane et al., 2008). Thus, our results replicate prior research exploring the performance of these tasks in children in this age range.

\section{Future Research}

Future studies could focus on different measures of executive function by considering different tasks. DTI methodologies and data analysis can be adjusted and alternative metrics such as mean diffusivity can be considered. In addition, future studies can report results from a control group of typically developing children to investigate whether the findings apply specifically to children with ADHD.

\section{Limitations}

Limitations in this study make it more difficult to argue that our findings generalize to understanding the association between SLFII white matter properties and executive function performance in young children with ADHD. As we already discussed, is that the sample size was reasonable but small. As mentioned, this makes it difficult to detect small effects, which may in fact be real. Therefore, we are at an increased risk of reporting what would be termed a "false negative" effect. That is, we are reporting no effect is present, but it may be the case that we do not have sufficient sample size to detect a real effect that may be present. 


\section{Acknowledgments}

We thank the members of the Developmental Cognitive Neuroscience Laboratory at FIU and other staff members for MRI acquisition, behavioral testing, research design, and writing the manuscript.

\section{References}

Alvarez, J. A., \& Emory, E. (2006). Executive function and the frontal lobes: a meta-analytic review. Neuropsychology review, 16(1), 17-42. https://doi.org/10.1007/s11065-006-9002-x

Berman, K. (1995). Physiological activation of a cortical network during performance of the Wisconsin Card Sorting Test: A positron emission tomography study. Neuropsychologia, 33(8). https://doi.org/10.1016/00283932(95)00035-2

Catani, M., \& Thiebaut de Schotten, M. (2008). A diffusion tensor imaging tractography atlas for virtual in vivo dissections. Cortex; a journal devoted to the study of the nervous system and behavior, 44(8), 1105-1132. https://doi.org/10.1016/j.cortex.2008.05.004

Eriksen, B.A., \& Eriksen, C.W. (1974). Effects of noise letters upon the identification of a target letter in a nonsearch task. Perception \& Psychophysics, 16, 143-149.

Fabiano, G. A., Pelham, W. E., Jr., Waschbusch, D. A., Gnagy, E. M., Lahey, B. B., Chronis, A. M., . . BurrowsMaclean, L. (2006). A practical measure of impairment: psychometric properties of the impairment rating scale in samples of children with attention deficit hyperactivity disorder and two school-based samples. J Clin Child Adolesc Psychol, 35(3), 369-385. https://doi:10.1207/s15374424jccp3503_3

Fischer, M., Moscovitch, M., \& Alain, C. (2020). A systematic review and meta-analysis of memory-guided attention: Frontal and parietal activation suggests involvement of fronto-parietal networks. WIREs Cognitive Science, 12(1). https://doi.org/10.1002/wcs.1546

Graziano, P. A., Slavec, J., Hart, K., Garcia, A., \& Pelham, W. E. (2014). Improving school readiness in preschoolers with behavior problems: Results from a summer treatment program. Journal of Psychopathology and Behavioral Assessment, 36, 555-569.

Kinoshita, M., Nakajima, R., Shinohara, H., Miyashita, K., Tanaka, S., Okita, H., Nakada, M., \& Hayashi, Y. (2016). Chronic spatial working memory deficit associated with the superior longitudinal fasciculus: a study using voxel-based lesion-symptom mapping and intraoperative direct stimulation in right prefrontal glioma surgery. Journal of Neurosurgery, 125(4), 1024-1032. https://doi.org/10.3171/2015.10.jns1591

Mullane, J. C., Corkum, P. V., Klein, R. M., \& McLaughlin, E. (2008). Interference Control in Children with and without ADHD: A Systematic Review of Flanker and Simon Task Performance. Child Neuropsychology, 15(4), 321-342. https://doi.org/10.1080/09297040802348028

Oeri, N., Voelke, A. E., \& Roebers, C. M. (2018). Inhibition and behavioral self-regulation: An inextricably linked couple in preschool years. Cognitive Development, 47. https://doi.org/10.1016/j.cogdev.2018.01.004 
Parlatini, V., Radua, J., Dell’ Acqua, F., Leslie, A., Simmons, A., Murphy, D. G., Catani, M., \& Thiebaut de Schotten, M. (2017). Functional segregation and integration within fronto-parietal networks. NeuroImage, 146, 367-375. https://doi.org/10.1016/j.neuroimage.2016.08.031

Pelham, W. E., Gnagy, E. M., Greenslade, K. E., \& Milich, R. (1992). Teacher ratings of DSM-III-R symptoms for the disruptive behavior disorders. Journal of the American Academy of Child \& Adolescent Psychiatry, 31(2), 210218.

Roberts, R. E., Anderson, E. J., \& Husain, M. (2010). Expert Cognitive Control and Individual Differences Associated with Frontal and Parietal White Matter Microstructure. Journal of Neuroscience, 30(50), 17063-17067. https://doi.org/10.1523/jneurosci.4879-10.2010

Schmahmann, J. D., \& Pandya, D. N. (2006). Fiber Pathways of the Brain. Oxford: Oxford University Press.

Smolker, H. R., Friedman, N. P., Hewitt, J. K., \& Banich, M. T. (2018). Neuroanatomical Correlates of the Unity and Diversity Model of Executive Function in Young Adults. Frontiers in Human Neuroscience, 12, 283-283. PubMed. https://doi.org/10.3389/fnhum.2018.00283

Wechsler, D. (2012). Wechsler Preschool and Primary Scale of Intelligence-Fourth edition technical and interpretive manual. In: San Antonio, TX: Pearson.

Zelazo, P. D., Muller, U., Frye, D., \& Marcovitch, S. (2003). I. The Development of Executive Function. Monographs of the Society for Research in Child Development, 68(3), 1-27. https://doi:10.1111/j.0037976x.2003.00261.x

Zelazo, P. D., Anderson, J. E., Richler, J., Wallner-Allen, K., Beaumont, J. L., \& Weintraub, S. (2013). Ii. Nih Toolbox Cognition Battery (Cb): Measuring Executive Function and Attention. Monographs of the Society for Research in Child Development,78(4), 16-33. https://doi:10.1111/mono.12032 